\title{
O consumo de selênio e sua relação com a manutenção da função cognitiva: uma revisão sistemática sobre humanos e animais
}

\author{
Consumption of selenium and its relation to maintenance of \\ cognitive function: a systematic review \\ of human and animal
}

\section{Consumo de selenio y su relación con el mantenimiento de la función cognitiva: una revisión sistemática de humanos y animales}

\author{
Brendon Stallen Lopes Nogueira ${ }^{1}$, Mariana Ducatti ${ }^{2}$, \\ Everton Horiquini-Barbosa ${ }^{3}$
}

1.Nutricionista, Faculdade Barretos, Barretos-SP, Brasil.

2.Psicóloga, Doutora em Ciências, Professora da Faculdade Barretos, Barretos-SP, Brasil.

3.Fisioterapeuta, Doutor em Ciências, Professor da Faculdade Barretos, Barretos-SP, Brasil.

\begin{abstract}
Resumo
Introdução. O processo de envelhecimento é marcado por alterações biológicas, psicológicas e sociais; e a depender da interação de tais variáveis nota-se a presença de patologias típicas de idosos. Uma preocupação relacionada ao envelhecimento é referente a perda das funções cognitivas, como memória, linguagem e aprendizagem. Contudo, diversos estudos têm indicado fatores de proteção cognitiva, sendo a alimentação um deles. Objetivo. Deste modo, o objetivo deste estudo foi investigar a relação entre selênio e a manutenção da função cognitiva. Método. Este estudo trata-se de uma revisão sistemática da literatura, realizada nas bases eletrônicas de dados PubMed, MedLine e Lilacs, utilizando a combinação de palavraschave específicas. Resultados. A busca resultou em 270 estudos, contudo, aplicado os critérios de inclusão e exclusão, selecionou-se oito artigos. Conclusão. Por meio dos artigos selecionados, observou-se que o consumo de selênio pode melhorar o sistema antioxidante e assim, apresentar efeitos positivos sobre a função cognitiva em idosos humanos. Os estudos com animais mostraram que a suplementação por selênio foi capaz de diminuir o estresse oxidativo e melhorar o aprendizado e o funcionamento da memória em testes comportamentais específicos. Em síntese, os estudos discutidos neste trabalho mostram uma alta relação do consumo de selênio e a melhora do desempenho cognitivo, tanto em humanos, quanto em animais.
\end{abstract}

Unitermos. Selênio; Idoso; Cognição; Memória; Aprendizagem

\begin{abstract}
Introduction. The aging process is marked by biological, psychological, and social changes; and depending on the interaction of such variables, the presence of pathologies typical of the elderly is noted. A concern related to aging is related to the loss of cognitive functions, such as memory, language, and learning. However, several studies have indicated cognitive protection factors, with food being one of them. Objective. Thus, the aim of this study was to investigate the relationship between selenium and the maintenance of cognitive function. Method. This study is a systematic review of the literature, carried out in the electronic databases PubMed, MedLine and Lilacs, using the combination of specific keywords. Results. The search resulted in 270 studies, however, applying the inclusion and exclusion criteria, eight articles were selected. Conclusion. Through the selected articles, it was observed that the consumption of selenium can improve the antioxidant system and, thus, present positive
\end{abstract}


effects on the cognitive function in elderly humans. Animal studies have shown that selenium supplementation was able to decrease oxidative stress and improve learning and memory function in specific behavioral tests. In summary, the studies discussed in this work show a high relation of selenium consumption and the improvement of cognitive performance, both in humans and in animals.

Keywords. Selenium; Aged; Cognition; Memory; Learning

\section{RESUMEN}

Introducción. El proceso de envejecimiento está marcado por cambios biológicos, psicológicos y sociales; y dependiendo de la interacción de tales variables, se nota la presencia de patologías propias del anciano. Una preocupación relacionada con el envejecimiento está relacionada con la pérdida de funciones cognitivas, como la memoria, el lenguaje y el aprendizaje. Sin embargo, varios estudios han indicado factores de protección cognitiva, siendo la alimentación uno de ellos. Objetivo. Por tanto, el objetivo de este estudio fue investigar la relación entre el selenio y el mantenimiento de la función cognitiva. Método. Este estudio es una revisión sistemática de la literatura, realizada en las bases de datos electrónicas PubMed, MedLine y Lilacs, utilizando la combinación de palabras clave específicas. Resultados. La búsqueda resultó en 270 estudios, sin embargo, aplicando los criterios de inclusión y exclusión, se seleccionaron ocho artículos. Conclusión. A través de los artículos seleccionados, se observó que el consumo de selenio puede mejorar el sistema antioxidante y, por tanto, presentar efectos positivos sobre la función cognitiva en humanos ancianos. Los estudios en animales han demostrado que la suplementación con selenio pudo disminuir el estrés oxidativo y mejorar la función de aprendizaje y memoria en pruebas de comportamiento específicas. En resumen, los estudios discutidos en este trabajo muestran una alta relación del consumo de selenio y la mejora del rendimiento cognitivo, tanto en humanos como en animales.

Palabras clave: Selenio; Ancianos; Cognición; Memoria; Aprendizaje

Trabalho realizado na Faculdade Barretos, Barretos-SP, Brasil

Endereço para correspondência: Everton Horiquini-Barbosa. Faculdade Barretos. Avenida C-12, 1555. Cristiano de Carvalho. CEP 14781-449. Barretos-SP, Brasil. E-mail: horiquini@unibarretos.com.br

\section{INTRODUÇÃO}

A mudança na estruturação demográfica, notada em todo o mundo, tem sido marcada pela diminuição da taxa de natalidade e aumento na expectativa de vida; o que ocasiona o aumento no número de idosos ${ }^{1,2}$. Estimativas indicam, por exemplo, que em 2050 a população mundial aumentará para aproximadamente 9,8 bilhões de pessoas, e deste, esperase que aproximadamente 2 bilhões sejam idosos, ou seja, tenham 60 anos ou mais ${ }^{1,2}$.

O processo de envelhecimento é contínuo e provocado por alterações morfológicas, fisiológicas, bioquímicas, 
psicológicas, cognitivas e comportamentais. Tais alterações podem ocasionar a diminuição gradual da capacidade física e cognitiva, assim como, maiores chances do desenvolvimento de patologias e a morte ${ }^{1,2}$.

Dentre as doenças típicas do envelhecimento, têm-se uma preocupação acentuada em relação aos déficits das funções cognitivas, em especial alterações de memória e aprendizagem ${ }^{3,4}$.

Uma alimentação adequada nessa fase da vida é extremamente importante para a manutenção das funções cognitivas. A manutenção a um padrão alimentar saudável com um consumo diário de frutas e hortaliças ricas em antioxidantes, reduzindo o consumo de ácidos graxos saturados e aumentando a ingestão de ácidos graxos insaturados demonstram ser a melhor opção para a prevenção na deterioração da memória ${ }^{5-7}$.

Os antioxidantes, por exemplos são substâncias fundamentais para a saúde cerebral, uma vez que sua ação pode evitar reações oxidativas e proteger os neurônios, 0 que favorece a manutenção da memória e a capacidade de aprendizagem ${ }^{8-10}$. De modo geral, alimentos in natura desempenham esse papel funcional, pois são ricos em compostos bioativos, como os flavonoides, vitamina $A$, vitamina C, vitamina E e o selênio (Se).

Por fim, o Se influencia o metabolismo de neurotransmissores e está relacionado com a capacidade antioxidante. $O$ consumo de Se é importante porque provoca o aumento da concentração sistêmica e a manutenção das 
funções cerebrais. Em relação a cognição, por exemplo, os níveis de Se diminuem à medida que as funções cognitivas ficam deficitárias; estudos indicam baixos níveis de Se em indivíduos com Doença de Alzheimer ${ }^{11}$. É importante ressaltar que um alimento rico em Se é a "castanha-doBrasil".

Em suma, considerando o aumento da expectativa de vida, o aumento da população idosa e consequentemente o aumento das doenças típicas do envelhecimento, torna-se importante estudos e práticas que promovam o envelhecimento saudável, auxiliem na estabilização de doenças do envelhecimento e promovam reabilitação das funções física e cognitivas comprometidas pelo envelhecimento. Assim, um campo importante de estudo está relacionado a nutrição e dieta, ou seja, estudo que relacionam a dieta com a manutenção das funções cognitivas. Deste modo, neste estudo buscou-se, por meio de uma revisão sistemática, investigar e discutir os efeitos da ingestão de Se em relação as suas propriedades antioxidantes para a preservação das funções cognitivas em idosos e da memória de ratos idosos.

\section{MÉTODO}

Foi realizada uma revisão sistemática de artigos científicos que analisou a relação entre o consumo de Se e a função cognitiva. A identificação dos artigos foi realizada nas bases eletrônicas de dados Pubmed, Medical Literature 
Analysis and Retrieval System Online (MEDLINE) e Literatura Latino-Americana e do Caribe em Ciências da Saúde (LILACS), por dois pesquisadores independentes (BSLN) e (EHB).

Em todas as bases de dados, a busca foi realizada utilizando os unitermos: "Selenium" e "Aged", juntamente com os unitermos que revezaram entre si: "Cognition", "Memory" e "Learning", obtendo-se 288 artigos.

Para a seleção dos artigos, foram aplicados critérios de inclusão e exclusão. Os critérios de inclusão foram: artigos em que o estudo fosse com animais do tipo roedor e/ou com seres humanos adultos; estudos relacionados à função cognitiva; estudos que utilizassem Se; artigos com tempo de publicação não superior aos últimos cinco anos. Critérios de exclusão: estudos que não fossem com animais do tipo roedores; estudos feitos com crianças; estudos que utilizassem o Se para outros fins; estudos publicados antes de 2014.

Inicialmente, os estudos foram selecionados a partir dos títulos dos artigos encontrados, seguido de uma segunda seleção por meio da leitura dos resumos e por fim, uma terceira seleção a partir da leitura do artigo na íntegra.

\section{RESULTADOS}

Seguindo a estratégia definida, a busca bibliográfica resultou em 270 artigos no total. Na base de dados do PUBMED, utilizando a combinação de unitermos "SELENIUM 
AND AGED AND LEARNING", foram encontrados 31 artigos. Com a combinação "SELENIUM AND AGED AND MEMORY" foram encontrados 42 artigos. $E$ por fim, combinando "SELENIUM AND AGED AND COGNITION" obteve-se 35 artigos.

No MEDLINE utilizou-se a mesma combinação de unitermos "SELENIUM AND AGED AND LEARNING", "SELENIUM AND AGED MEMORY" e "SELENIUM AND AGED AND COGNITION", com isso, foram encontrados 17, 40, 32 artigos, respectivamente.

No LILACS, utilizando os unitermos "SELENIUM AND AGED AND LEARNING", foram encontrados 16 artigos e com a combinação "SELENIUM AND AGED AND MEMORY", foram encontrados 24 artigos. Por último, utilizando a combinação "SELENIUM AND AGED AND COGNITION", foram encontrados 33 artigos.

Numa primeira análise, verificou-se que 168 artigos não estavam relacionados com o tema proposto e por isso foram excluídos, restando, portanto, 102 artigos. Por meio de uma análise mais aprofundada dos 102 artigos selecionados, à luz dos objetivos do estudo, restaram 53 artigos. Após a remoção de duplicatas (40 excluídos) e de artigos anteriores a data definida (5 excluídos), oito artigos constituíram o grupo de publicações definitivamente considerado para a análise proposta. A Figura 1 ilustra as etapas de seleção dos artigos. Os oito estudos que atenderam aos critérios metodológicos adotados para este trabalho são descritos a seguir. 


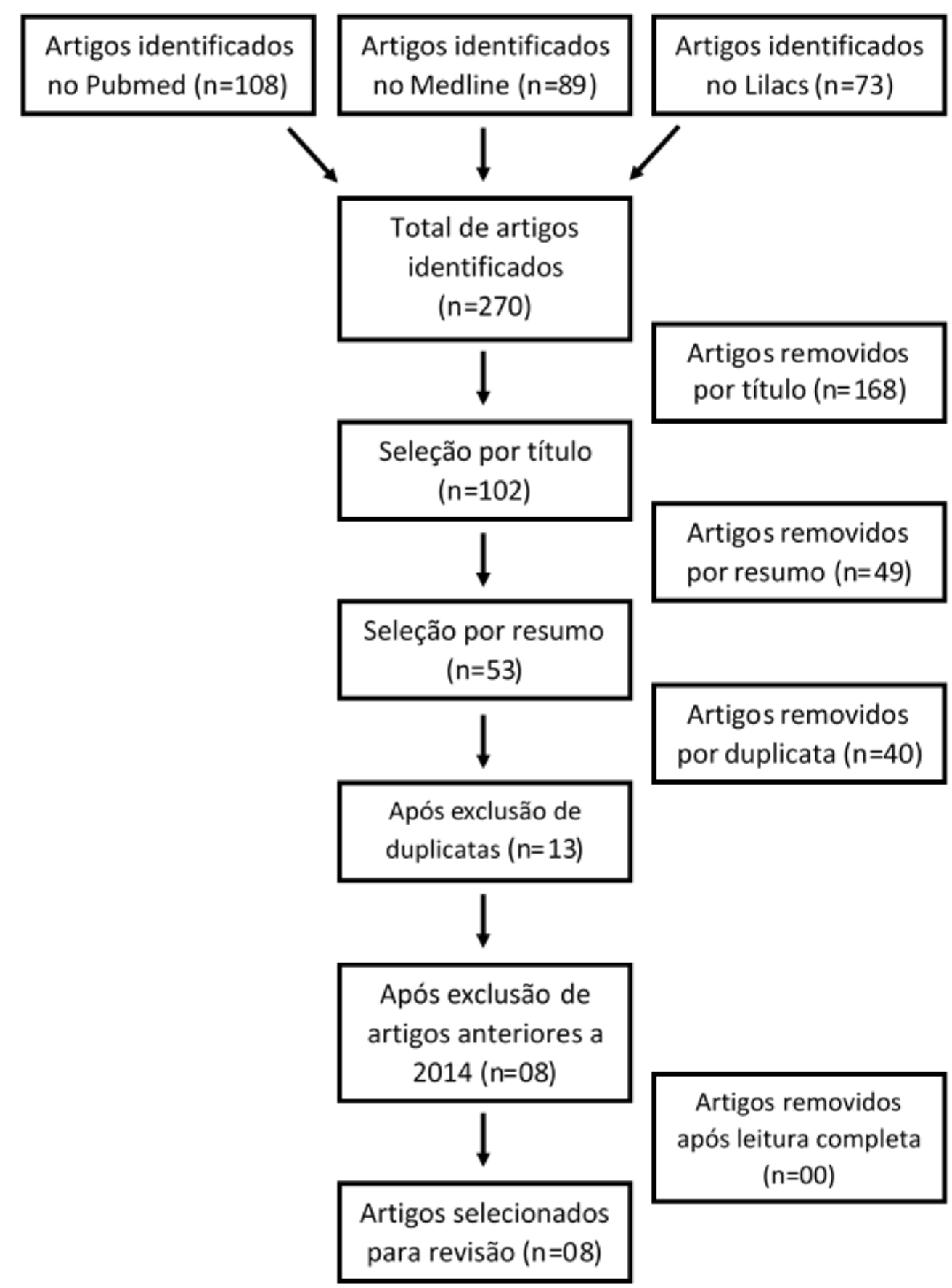

Figura 1. Fluxograma do passo-a-passo para obtenção do total de sete artigos que foram incluídos na revisão, após busca e utilização de critérios estabelecidos de inclusão e exclusão.

Artigo 1.12 O estudo teve como objetivo determinar a quantidade de Se em idosos com Doença de Alzheimer (DA) ou Declínio Cognitivo Leve (DCL) e compará-los com idosos saudáveis (GC). O grupo DA foi formado por 27 idosos com diagnosticados pela doença, o grupo DCL por 31 
participantes e GC por 28 idosos saudáveis. Foi realizada avaliação dietética utilizando registro alimentar não consecutivo, sete dias antes da coleta da amostra de sangue. A concentração de Se foi determinada em amostras de plasma e eritrócito usando espectroscopia de absorção atômica. Os resultados indicaram que a) não houve diferença de gêneros entre os grupos; b) a média de carboidrato ingerido pelo grupo DA foi mais alta comparada aos demais grupos, enquanto a quantidade de proteína e lipídio se mostrou mais baixa; c) os participantes dos três grupos, principalmente o grupo de idosos com DA, apresentaram ingestão inadequada de Se. De acordo com a concentração de Se no plasma sanguíneo e nos eritrócitos, o grupo DA apresentou o menor nível, muito embora, os outros grupos apresentaram nível abaixo do considerado aceitável. Com isso, é possível notar que os níveis de Se plasmático e nos eritrócitos tendem a diminuir juntamente com a diminuição da função cognitiva. Assim, a ingestão deficiente de Se é decorrente não somente da deficiência aguda, mas também crônica. O Se possui capacidade antioxidante, reduz a inflamação e marcadores de estresse oxidativo. Deste modo, o estudo conclui que a deficiência de Se pode contribuir para o declínio cognitivo em idosos, e que o consumo de alimentos ricos em Se, como a Castanha-do-Brasil, deve ser encorajado para restaurar o nível de Se plasmático e melhorar o sistema antioxidante. 
Artigo 2. ${ }^{13} \mathrm{O}$ objetivo deste estudo foi investigar se 0 consumo de Castanha-do-Brasil, melhor fonte alimentar de $\mathrm{Se}$, melhora a função cognitiva em idosos. $\mathrm{O}$ estudo foi feito com 31 participantes com DCL, acima de 60 anos e livres de quaisquer outras alterações neurológicas ou doenças psiquiátricas. Os participantes passaram por uma bateria de avaliações neuropsicológicas, que incluíam testes de atenção, memoria, linguagem, função visuo-espacial e funções executivas. Após os testes, o nível de Se e de radicais livres no plasma foram mensurados. Os participantes, então, foram divididos em 2 grupos, o grupo controle e o tratado. O grupo controle foi instruído a se alimentar normalmente e evitar o consumo de Castanha-doBrasil durante os 6 meses de estudo, enquanto o grupo tratado recebeu a instrução para consumir 1 Castanha-doBrasil por dia. Apenas 11 e 9 indivíduos concluíram o estudo, no grupo tratado e controle, respectivamente. Os resultados indicaram a) aumento significativo no nível de Se plasmático e melhora no sistema antioxidante no grupo tratado; b) todos os indivíduos do grupo controle apresentaram níveis abaixo da linha de corte aceitável; c) o desempenho cognitivo inicial não apresentou diferenças entre os grupos, mas após 6 meses de experimento, d) a fluência verbal e a construção de frases apresentaram melhora significativa no grupo que consumiu a Castanha-do-Brasil por 6 meses. Os dados sugerem que o consumo diário de uma castanha-doBrasil restaura a deficiência de Se. Há indicações de que a suplementação da castanha em idosos com DCL pode ter 
efeitos positivos em algumas funções cognitivas, por exemplo, fluência verbal, semântica e construção de frase.

Artigo 3. ${ }^{14} \mathrm{O}$ objetivo deste estudo foi investigar os efeitos do exercício físico e uma dieta suplementada com disseleneto de difenila envolvidos na neuroproteção do hipocampo de ratos idosos. Para o experimento, foram utilizados ratos machos, tipo wistar com 4 meses de idade (adultos) ou 27 meses idade (idosos). Os animais foram divididos e 5 grupos (nove animais por grupo): I) grupo controle adulto - ratos adultos sedentários alimentados com dieta comercial padrão. II) grupo controle idoso - ratos idosos sedentários alimentados com dieta comercial padrão. III) grupo ( $\mathrm{PhSe}) 2$ - ratos idosos sedentários alimentados com dieta suplementada com $1 \mathrm{ppm}$ de (PhSe)2. IV) grupo exercício - ratos idosos submetidos ao protocolo de treinamento de natação e tratados com uma dieta comercial padrão. V) grupo exercício/(PhSe)2 - ratos idosos submetidos ao protocolo de treinamento de natação e alimentados com dieta suplementada com 1 ppm de (PhSe)2. Os animais passaram por processo de adaptação a natação e após a adaptação, foram submetidos ao treinamento de 20 minutos por dia e foram alimentados com ração padrão ou com ração suplementada com 1 ppm de (PhSe)2 durante 4 semanas. Os animais foram submetidos aos testes comportamentais de memória, reconhecimento de tarefas e localização de objetos. Após os testes comportamentais os ratos foram eutanasiados, os cérebros 
coletados para analisar o hipocampo. Os resultados indicam que a) o exercício de natação associado à uma dieta suplementada com ( $\mathrm{PhSe} 2$ modulou os níveis de proteínas envolvidas em apoptose e b) proteínas envolvidas aos processos inflamatórios no hipocampo de ratos idosos. Em suma, o estudo indica que o exercício físico e dieta suplementada com (PhSe)2 promovem uma ação neuroprotetiva no hipocampo de ratos idosos.

Artigo 4. ${ }^{15} \mathrm{O}$ objetivo deste estudo foi determinar os efeitos de uma dieta suplementada com (PhSe)2 e exercício de natação para a memória de ratos de meia idade. Para este estudo foram usados ratos wistar machos, adultos (3 meses de idade) e de meia idade (12 meses de idade). Os animais foram separados em cinco grupos: I) grupo controle adulto - animais adultos que não nadaram; II) grupo controle animais de meia-idade que não nadaram; III) grupo exercício - animais que nadaram; IV) grupo (PhSe)2 animais que não nadaram e foram alimentados com dieta suplementado com 1ppm de (PhSe)2; V) exercício + (PhSe)2 - animais que nadaram e foram alimentados com dieta suplementado com 1ppm de (PhSe)2. Estes animais foram mantidos em gaiolas separadas com livre acesso a comida e água da torneira. Os ratos passaram por processo de adaptação a natação, após a adaptação, foram submetidos ao treinamento de 20 minutos por dia e foram alimentados com ração padrão ou com ração suplementada com 1ppm de (PhSe)2 durante 4 semanas. Os animais foram submetidos 
aos testes comportamentais de reconhecimento de objetos e localização de objetos. Após todos os testes comportamentais, todos os ratos foram eutanasiados e os cérebros foram coletados e amostras de hipocampo separadas. Os resultados indicam que a) a associação entre dieta suplementada com (PhSe)2 e exercícios de natação melhorou os parâmetros de reconhecimento de objeto e b) localização de objetos em ratos de meia-idade. O (PhSe)2 é um sintético simples, composto de organosSe, que apresenta inúmeros benefícios para a saúde quando utilizado em doses farmacológicas. Neste estudo, a dieta suplementada com (PhSe)2 foi eficaz em melhorar a memória de ratos de meia-idade em todas as tarefas. Em conclusão, este estudo demonstrou que o exercício de natação associado à dieta suplementada com (PhSe)2 pode melhorar o aprendizado e funcionamento da memória.

Artigo 5. ${ }^{16}$ Este estudo teve como objetivo investigar se uma dieta suplementada com (PhSe)2 e exercício de natação para a memória de ratos. O estudo utilizou ratos Wistar com 4 meses de idade (adultos) ou 27 meses idade (idosos). Os animais foram divididos em 1 grupo de animais adultos e 4 grupos de animais idosos. I) grupo controle adulto - ratos adultos sedentários alimentados com dieta comercial padrão. II) grupo controle idoso - ratos idosos sedentários alimentados com dieta comercial padrão. III) grupo (PhSe)2 - ratos idosos sedentários alimentados com dieta suplementada com 1 ppm de (PhSe)2. IV) grupo exercício - 
ratos idosos submetidos ao protocolo de treinamento de natação e tratados com uma dieta comercial padrão. V) grupo exercício/(PhSe)2 - ratos idosos submetidos ao protocolo de treinamento de natação e alimentados com dieta suplementada com 1 ppm de (PhSe)2. Os animais passaram por período de adaptação a natação e após a adaptação, foram submetidos ao treinamento de 20 minutos por dia com carga de $3 \%$ do peso corporal e foram alimentados com ração padrão ou com ração suplementada com 1 ppm de (PhSe)2 durante 4 semanas. Os animais foram submetidos aos testes comportamentais de memória como reconhecimento de tarefas, localização de objetos e o campo aberto. Após os testes comportamentais os ratos foram eutanasiados, os cérebros coletados para analisar 0 hipocampo. Os resultados demonstraram que a) a ingestão de uma dieta suplementada com (PhSe)2 e o exercício de natação foi eficaz para melhorar a memória de curto e longo prazo; b) aprendizagem espacial; c) aumentando os níveis de fosforilação da proteína de ligação ao elemento de resposta do AMPc (CREB) no hipocampo dos ratos idosos. Deste modo, este estudo também fornece evidências de que a dieta suplementada com (PhSe)2 facilita a memória de ratos idosos, modulando os níveis de AMPc e estimulando a fosforilação de CREB.

Artigo 6. ${ }^{17}$ Este estudo teve como objetivo investigar se 0 Se tem efeitos protetores sobre a demência induzida por Escopolamina (SCOP) e sobre o dano oxidativo em ratos 
idosos. O estudo utilizou 32 ratos Wistar albinos com idade entre 18 - 24 meses. Os ratos idosos foram igualmente divididos em quatro grupos da seguinte forma: I) Grupo controle - sem comprometimento da memória, não foi suplementado e receberam solução salina a 0,9\% para injeção peritoneal por 14 dias. II) Grupo demência - a demência foi induzida por injeção de SCOP $(1 \mathrm{mg} / \mathrm{kg}$ durante 21 dias). III) Grupo Se - os ratos receberam injeção de $1,5 \mathrm{mg} / \mathrm{kg}$ de Se, em dias intervalados por 14 dias, totalizando 7 doses. IV) grupo demência + Se - os ratos receberam injeção de Se (igual ao grupo III) após a indução da demência (igual ao grupo II). Os ratos foram eutanasiados, as amostras de sangue e cérebro (lobo temporal) foram coletadas. No presente estudo foi observado a) uma diminuição dos níveis de peroxidação lipídica no cérebro e eritrócitos nos ratos com demência experimental e tratados com Se. Este estudo também fornece evidências de que o Se é capaz de proteger o cérebro e o eritrócito contra a toxicidade oxidativa induzida pela demência experimental. $O$ tratamento com Se regulou os níveis de peroxidação lipídica no cérebro, sangue e eritrócitos de ratos com demência experimental, sugerindo que o Se desempenha uma função bioquímica essencial, retardando os processos oxidativos e a apoptose, servindo como um potencial antioxidante.

Artigo 7.18 $\mathrm{O}$ objetivo deste estudo foi testar os efeitos do tratamento com Se sobre a apoptose, estresse oxidativo e 
influxo de $\mathrm{Ca}^{+}$por meio dos canais TRPM2 e TRPV1 no gânglio da raiz dorsal e no hipocampo de ratos idosos induzidos por escopolamina (SCOP). O estudo utilizou 32 ratos Wistar albinos, com idade entre 18 - 24 meses. Os ratos foram alimentados com comida comercial e foi permitido beber água a vontade. Os ratos idosos foram divididos em quatro grupos, sendo oito animais por grupo: I) grupo controle - recebeu injeção de $1 \mathrm{ml}$ de solução fisiológica salina $(0,9 \% \mathrm{NaCl})$ por dia. II) grupo SCOP recebeu injeção de SCOP (1mg/kg durante 21 dias). III) grupo Se - recebeu injeção de $1,5 \mathrm{mg} / \mathrm{kg}$ de Se por 14 dias. IV) grupo SCOP + Se - recebeu SCOP tratado com Se (SCOP + Se). O grupo SCOP + Se - recebeu injeção de SCOP por 3 semanas e então recebeu injeção de Se por 14 dias. Os animais foram testados para verificar a memória e após os testes comportamentais, os ratos foram eutanasiados e amostras do hipocampo foram coletadas. No modelo experimental de demência induzida por escopolamina, os canais TRPM2 e TRPV1 estão envolvidos na morte neuronal que é induzida por entrada de $\mathrm{Ca}^{+}$nos neurônios do hipocampo e do gânglio da raiz dorsal; entretanto, o tratamento com Se reduziu atividade dos canais e isso pode explicar sua atividade neuroprotetora contra a apoptose. O tratamento com Se também gerou melhora nos testes de memória. Esses achados têm significado particular e podem fornecer uma explicação para morte neuronal induzida pelo SCOP e para melhora da memória devido ao Se por meio de vias endógenas de estresse oxidativo. Parece que os canais 
TRPM2 e TRPV1 podem se tornar importantes alvos farmacológicos no tratamento da oxidação induzida.

Artigo 8. ${ }^{19}$ Neste estudo, o efeito terapêutico e os mecanismos subjacentes da selenometionina (Se-Met) na patologia da tau foi explorada em camundongos com 8 meses de idade, transgênicos para Doença de Alzheimer (DA). Os camundongos DA do grupo experimental ( $n 12$; seis machos e seis fêmeas; 8 meses de idade) foram tratados com Se-Met $6 \mathrm{~g} / \mathrm{ml}$ em água potável por 12 semanas. Os camundongos controle ( $n=12$ por grupo; seis machos e seis fêmeas) receberam água potável normal. Após o tratamento com Se-Met durante 12 semanas foram realizados testes comportamentais para verificar a memória, e então os camundongos foram eutanasiados e seus cérebros foram rapidamente removidos e análisados. Foi demonstrado, pela primeira vez, que a) o tratamento com Se-Met reduziu a hiperfosforilação da tau, além disso, b) o Se-Met melhorou o a memória espacial em camundongos DA. Em conclusão, o presente estudo demonstrou que Se-Met reduz a hiperfosforilação da tau, e melhora a performance em teste de memória.

\section{DISCUSSÃO}

Neste estudo, investigou-se e discutiu-se os efeitos da ingestão de Se, em relação as suas propriedades 
antioxidantes para a preservação das funções cognitivas em idosos e da memória de ratos idosos. Foi utilizado o modelo de revisão sistemática para buscar artigos relacionados ao tema, que abordassem o Se como forma de prevenção e/ou tratamento de doenças degenerativas que afetam o sistema cognitivo. Os principais resultados encontrados foram em relação a a) o número de estudos com animais e humanos; b) metodologia utilizada; c) intervenção escolhida; e d) o resultado encontrado pelos artigos selecionados. A seguir, há considerações de cada um desses achados.

\section{Efeito do selênio em humanos}

O primeiro ponto importante a se destacar na busca de estudos que investigam e discutem os efeitos da utilização de Se e sua relação com função cognitiva em idosos é que poucos estudos se ocupam deste objetivo científico, e deste modo, se mostrando um campo de pesquisa pouco explorado mas que merece atenção dos pesquisadores. Além disso, é sabido que o nível de selênio é altamente variável nos alimentos, mas a Castanha-do-Brasil (Bertholletia excelsa) é a fonte alimentar mais rica em selênio, e por se tratar de um alimento originário do Brasil ${ }^{13}$, se faz ainda mais relevante que pesquisadores brasileiros se voltem para pesquisas nesta área de atuação.

Foram selecionados apenas 2 estudos que verificaram os efeitos do Se e sua relação com função cognitiva em idosos e um achado que se destaca entre os artigos selecionados é a quantidade de sujeitos de pesquisa 
selecionados em cada artigo. Foi possível observar que no artigo $1^{12}$ foram utilizados 27 sujeitos com provável DA, 31 sujeitos diagnosticados com CCL e 28 sujeitos saudáveis; no artigo $2^{13}$ foram utilizados 31 sujeitos com CCL.

Neste estudo, os sujeitos com provável DA atendem os critérios da National Institute of Neurological and Communicative Disorders and Stroke and the Alzheimer's Disease and Related Disorders Association (NINCDS-ADRDA) que estão entre os critérios mais utilizados e aceitos para o diagnóstico desta condição ${ }^{12}$. Segundo os critérios da NINCDS-ADRDA é possível o diagnóstico de DA definitiva, provável, possível ou improvável. Esses critérios requerem que a presença de alteração cognitiva e uma síndrome demencial sejam confirmadas por testes neuropsicológicos para um diagnóstico clínico de DA possível ou provável; enquanto para o diagnóstico definitivo, há necessidade de confirmação histopatológica ${ }^{3,20}$.

Todos os sujeitos com CCL incluídos nos artigos $1^{12} \mathrm{e}$ $2^{13}$ foram diagnosticados por meio dos critérios da International Working Group on Mild Cognitive Impairment. Tais critérios são: a) a pessoa não tem funcionamento cognitivo norma, porém não tem demência; b) há evidência de deterioração cognitiva identificada por relato e conjunto com déficits cognitivos; e c) as atividades da vida diária são preservadas e as funções instrumentais complexas estão preservadas ou minimamente prejudicadas ${ }^{21}$.

Os artigos incluíram indivíduos de ambos os sexos, com aproximadamente 60 anos e distribuídos cegamente nos 
grupos estudados. A escolha do público pesquisado faz sentido, visto que o declínio cognitivo está relacionado com o aumento da idade e pelo aumento de radicais livres no cérebro $3,4,6,7$.

Também é importante observar que em ambos os estudos foi utilizada a espectrometria de absorção atômica acoplada à geração de hidretos para a análise da dosagem de Se plasmático. Em ambos os estudos selecionados, foram coletados sangue dos pacientes em jejum e o material passou por centrifugação, lavagem com soro e foi criopreservado; e a partir do plasma e eritrócito foi possível analisar a quantidade de Se e espécie reativa de oxigênio. A espectrometria de absorção atômica acoplada à geração de hidretos é um poderoso e sensível instrumento utilizado para análise de metais como o Se ou arsênio, fazendo com que a escolha da técnica seja adequada para os estudos, aumentando a fidedignidade dos resultados ${ }^{22}$. Entretanto, o método é de alto custo e limita sua utilização.

Ainda sobre a metodologia dos estudos selecionados, 0 artigo $2^{13}$ iniciou os procedimentos com 31 pacientes selecionados, entretanto, por ter sido um estudo de longo prazo, alguns pacientes não foram capazes de conclui-lo, deste modo, apenas 20 participantes chegaram ao final do estudo. Os autores destacaram os motivos da exclusão dos pacientes, sendo eles: mudança de endereço, conduta inadequada, infarto do miocárdio ou pacientes que tomaram multivitamínico que possui $\mathrm{Se}$ e isso poderia interferir o resultado final do estudo. Porém, apesar da diminuição no 
número de participantes, os autores comprovaram suas hipóteses.

Em relação a intervenção escolhida, houve uma divergência em relação ao tempo de ingestão da castanhado-Brasil, sendo que no artigo $2^{13}$ a intervenção escolhida é o consumo diário de uma castanha-do-Brasil durante 6 meses (24 semanas), enquanto o estudo de Behr mostra que o consumo de uma castanha-do-Brasil durante 12 semanas é suficiente para aumentar as concentrações de Se no plasma e eritrócito ${ }^{23}$. Ao comparar-se os resultados de ambos os artigos, observa-se uma diferença de tempo de intervenção e, mas independentemente disto, a capacidade do Se suprir as necessidades cognitivas dos idosos.

Deste modo, os resultados foram consensuais quanto aos benefícios do Se para a população idosa, possuindo como principais achados, a melhora o sistema antioxidante; protegendo as células contra danos causados por espécie reativa de oxigênio; aumentando a proporção dos níveis de lipoproteína de alta densidade (HDL); e inibindo a lipoperoxidação.

Por último cabe ressaltar que o número de estudos clínicos encontrados que abordam a utilização do Se para melhora da função cognitiva é pequeno, além disso apresentam metodologias e tempo de intervenção variados, deste modo, limitando a possibilidade de comparações de resultados. Portanto, se faz necessário mais estudos para elucidar os questionamentos existentes nesta área de pesquisa. 


\section{Efeito do selênio em animais}

A maior parte dos artigos encontrados com o método de busca aqui utilizado foram estudos experimentais que utilizavam animais como ratos, camundongos e cobaias. Deste modo, após os critérios de inclusão e exclusão, apenas 6 artigos experimentais constituíram o grupo de publicações definitivamente considerado para a análise proposta.

Os artigos $3^{14}$ e $4^{15}$ apresentam similaridades na metodologia utilizada pois os dois estudos buscam a relação do exercício físico aeróbico associado à dieta suplementada com disseleneto de difenila (PhSe)2. Contudo, vale observar alguns pontos importantes como o número de animais utilizados para o estudo, sendo que o artigo $3^{14}$ utilizou um total de 45 ratos para o estudo, enquanto no artigo $4^{15}$ não ficou explicito a quantidade de ratos utilizados para a pesquisa. Entretanto, as características dos animais são similares, no artigo $3^{14}$ foram utilizados ratos do tipo Wistar, machos, adultos (4 meses) e idosos ( 27 meses) enquanto no artigo $4^{15}$, foram utilizados ratos do tipo Wistar, machos, adultos ( 3 meses) e de meia idade (12 meses).

Os ratos são os animais mais utilizados em pesquisa, seguidos pela utilização de camundongos, coelhos, cães, porcos e primatas ${ }^{24}$. Outro fato interessante é a relação entre a idade dos ratos e de humanos, na idade adulta, a cada mês do rato é aproximadamente equivalente a 2,5 anos humanos ${ }^{25}$. Além disso, descrevem que ratos com 12 e 30 meses, são aproximadamente equivalentes a humanos com 30 e 75 anos de idade, indicando que os animais utilizados 
no artigo $3^{14}$ e $4^{15}$ equivalem a humanos de meia idade e idosos.

Em ambos os estudos, os ratos foram separados em cinco grupos, sendo: um grupo controle; um grupo adulto; um grupo sedentário suplementado com (PhSe)2; um grupo de exercício; um grupo submetido a natação e dieta suplementada com 1ppm de (PhSe)2. Além disso, os artigos são originários do mesmo grupo de pesquisa, o que pode levar ao uso de animais com características especificas do interesse do grupo.

Outro ponto a se destacar é em relação ao tempo de adaptação de treinos adotados nos estudos. No artigo $3^{14}$, os animais foram submetidos a um período de 20 minutos durante 5 dias, enquanto no artigo $4{ }^{15}$, os animais foram submetidos a um período de 20 minutos durante uma semana, não deixando claro se os animais foram submetidos a 5 ou 7 dias de adaptação. Após o período de adaptação à natação, os ratos realizaram o protocolo de exercício com carga, sendo maior no artigo $4^{15}$ ( $3 \%$ do peso corporal) comparado ao artigo $3^{14}$ ( $1 \%$ do peso corporal), por outro lado, o tempo de duração foram iguais em ambos (20 minutos/dias por 4 semanas). Segundo os pesquisadores dos artigos $3^{14}$ e $4^{15}$, o protocolo utilizado foi desenvolvido por Kiran et al (2004) ${ }^{26}$, que originalmente utiliza um protocolo de 5 dias de adaptação e 2, 3 ou $5 \%$ do peso corporal dos animais como carga.

Ainda sobre o protocolo de experimental, é válido observar o período em que os ratos foram submetidos aos 
treinamentos sendo que no artigo $3^{14}$, o treinamento foi realizado a partir das 9 a.m., enquanto que no outro trabalho os animais realizaram o treinamento a partir das 13 p.m., muito parecido com o protocolo original que inicia 0 treinamento as 10 a.m.. Neste ponto, é necessário relatar que estudos indicam que ratos possuem um ciclo claro/escuro e que é dependente da idade, portanto, ratos jovens de 3 e 4 meses de idade podem apresentar um ritmo circadiano diferente do de ratos de 12 e 27 meses de idade 27 . Deste modo, parece que os pesquisadores não levaram em conta esta característica etária dos animais, e que seria interessante a discussão sobre este ponto específico ou mesmo a comparação entre diferentes períodos de treinamento.

Ao final das 4 semanas de treinamento, 24 horas após o último dia de treinamento, os ratos foram submetidos aos testes comportamentais de memória e reconhecimento e localização de objetos. No artigo $3^{14}, 4^{15}$ e $5^{16}$ os ratos realizaram testes de memória de curta e longa duração, e todos os protocolos apresentaram resultados satisfatórios sugerindo que a dieta suplementada com (PhSe)2, associada ao exercício pode trazer benefícios em relação a memória e aprendizagem dos animais. Estudos prévios também demonstram que a dieta suplementada com (PhSe)2 possui propriedades que melhoram a memória de ratos através de testes como labirinto em $\mathrm{Y}^{28}$, o labirinto aquático de Morris ${ }^{29}$ e o teste de reconhecimento de objetos ${ }^{30}$. Evidências indicam que o ( $\mathrm{PhSe}) 2$ pode atravessar a barreira hemato-encefálica 
e aumentar o nível de Se no cérebro ${ }^{31}$ e que isso seria uma possível explicação para a melhora do memória.

Ainda nos artigos $3^{14}, 4^{15}$ e $5^{16}$, após os testes comportamentais, os animais foram eutanasiados, os seus cérebros coletados, os hipocampos foram preparados para análise de Western Blot. Apesar de adotarem o mesmo ensaio, as metodologias foram distintas.

Ainda em relação aos resultados, os autores do artigo $3^{14}$ indicam que a combinação de exercício e a dieta suplementada, modula os níveis de proteínas envolvidas na apoptose e processos inflamatórios no hipocampo de ratos idosos, corroborando os achados do artigo $4^{15}$ e $5^{16}$ que também mostra em seus resultados que o exercício associado a dieta suplementada melhorou todos os parâmetros de memória avaliados.

Em suma, os resultados dos artigos $3^{14}, 4^{15}$ e $5^{16}$ convergem entre si, trazendo ao leitor novas perspectivas, indicando que exercício associado a uma dieta suplementada com (PhSe)2 pode diminuir ou retardar os efeitos negativos na cognição por diminuir os níveis séricos de citocinas próinflamatórias, além de modular a neuroproteção.

O objetivo dos estudos $6^{17}$ e $7^{18}$ foi analisar a intervenção com o uso de Se e investigar as suas propriedades antioxidantes, anti-inflamatória, no combate à apoptose, oxidação celular e inflamação das células de ratos com demência induzida por SCOP. A SCOP é um antagonista dos receptores colinérgicos muscarínicos e serve como uma 
ferramenta farmacológica muito utilizada em modelos animais de alterações de memória ${ }^{32}$.

O primeiro ponto a ser observado foi à metodologia utilizada por estes estudos, que são muito semelhantes. Os estudos utilizaram 32 ratos com idade entre 18 - 24 meses, com peso de $320 \pm 30 \mathrm{~g}$. Os ratos foram submetidos à tarefa de labirinto radial por 14 dias antes de dar início aos testes, como habituação dos animais. Em seguida, foram distribuídos em 4 grupos, sendo: grupo controle; grupo demência; grupo Se; e um grupo demência tratado com Se. Ao final dos testes, todos os ratos foram mortos $e$ decapitados. Porém os estudos apresentaram diferentes condutas relacionadas à metodologia. $\mathrm{O}$ artigo $6^{17}$ realizou a coleta de sangue para análise do plasma e eritrócito além da coleta do cérebro para análise do lobo temporal. Enquanto isso, os autores do artigo $7^{18}$ coletaram o gânglio da raiz dorsal e o hipocampo para análise. Apesar das diferenças, foi possível observar em ambos, que a indução de demência com a SCOP aumenta os níveis de espécies reativas de oxigênio, aumentando o estresse oxidativo, a inflamação e aumenta os níveis da apoptose celular. Uma das hipóteses mais aceitas para as alterações de memória está relacionada disfunção mitocondrial e estresse oxidativo como eventos primários no surgimento da doença. ${ }^{33}$

Nos dois estudos, $6^{17}$ e $7^{18}$, os autores puderam observar em seus resultados, que no grupo demência tratado com Se, a suplementação foi capaz de diminuir a peroxidação lipídica no cérebro e eritrócitos. A administração 
de Se pode prevenir a neurodegeneração, bem como proteger o cérebro do dano causado pelo SCOP, pode melhorar a capacidade antioxidante, prevenindo a lesão oxidativa causada pela demência. Um alto nível de citocinas pró-inflamatórias, incluindo as interleucinas, estão presentes no cérebro de pacientes com demência e levam a alteração das funções neuroniais por meio do aumento do estresse oxidativo ${ }^{34}$, entretanto, foi demonstrado no artigo 6 que o Se diminui o nível de interleucinas no plasma sanguíneo por meio do sistema antioxidante.

Por último, o artigo $8^{19}$, teve como objetivo investigar o efeito terapêutico e os benefícios de selenometionina (SeMet) na patologia da tau. Diferente dos demais estudos feitos com ratos do tipo Wistar, este foi realizado com camundongos de 3xTg-AD (quarta geração), pois estes expressam o gene humano APPswe e tauP301L e o gene PS1M146V mutante. Foram utilizados 24 ratos, sendo 12 fêmeas e 12 machos com 8 meses de idade. Em seguida os ratos foram separados em dois grupos, sendo o primeiro grupo tratado com Se-Met $6 \mathrm{~g} / \mathrm{ml}$ em água potável por 12 semanas, enquanto o segundo grupo recebeu água potável normal. Semelhante aos artigos $6^{17}$ e $7^{18}$, os ratos do artigo 8 foram submetidos a um teste labirinto aquático que consiste em medir o tempo necessário para os ratos encontrarem a plataforma de saída ${ }^{35}$. Deste modo, a eficácia terapêutica da Se-Met foi constatada por meio da melhora observada no grupo tratado, com uma latência menor para 
encontrar a plataforma durante os treinos ou após $24 \mathrm{~h}$ e $72 \mathrm{~h}$ após a finalização do treino.

Após a coleta dos testes comportamentais, os ratos foram eutanasiados e seus cérebros foram removidos. 0 hemisfério esquerdo foi imerso em paraformaldeído, desidratado, limpo e cortado em pequenas fatias e a análise histopatológica com microscopia de fluorescência do hipocampo e região cortical revelou que os camundongos tratados com Se-Met mostraram marcadamente menor densidade de imunocoloração de tau total comparado aos ratos do grupo controle. O hemisfério direito foi dissecado em amostras de hipocampo e cortical, congelado em nitrogênio líquido e criopreservado e os resultados demostraram, por meio de técnica de Western Blot que a SeMet reduz a hiperfosforilação de tau. Song et al. $(2014)^{36}$, relatam que a Se-Met possui efeitos bioquímicos, contribuindo com a resistência à danos oxidativos devido a potente ação das selenoproteínas e selenoenzimas.

Analisando todos os estudos em conjunto, observa-se que os trabalhos possuem metodologias distintas como a espécie estudada, o modelo de indução dos danos à memória, o composto utilizado para tratamento e o tempo aplicado à pesquisa. Entretanto, quando comparados, foi possível notar uma linha de seguimento das pesquisas, um estudo parece complementar o outro. Os autores tiveram pontos de partida distintos entre si, porém, todos obtiveram a mesma conclusão geral: o Se é um micronutriente essencial que desempenha papel fundamental nas reações 
inflamatórias, atuando no sistema imune e protegendo as células contra danos oxidativos causados por espécies reativas de oxigênio.

\section{CONCLUSÃO}

Conclui-se que, a ingestão adequada de Selênio auxilia na manutenção das funções cerebrais. Em síntese, os estudos discutidos neste trabalho mostram uma alta relação do consumo de Se e a melhora do desempenho cognitivo, tanto em humanos, quanto em animais.

\section{REFERÊNCIAS}

1.Closs VE, Schwanke CHA. A evolução do índice de envelhecimento no Brasil, nas suas regiões e unidades federativas no período de 1970 a 2010. Rev Bras Geri Gerontol 2012;15:443-58. https://doi.org/10.1590/S1809-98232012000300006

2. World Halth Organization. Global Strategy and Action Plan on Ageing and Health (2016-2020). Geneva (acessado em 2020). Disponível em: https://www.who.int/ageing/global-strategy/en/

3.American Psychiatric Association. DSM-5: Manual diagnóstico e estatístico de transtornos mentais. Porto Alegre: Artmed Editora. 2014, p992.

4.Radanovic M, Stella F, Forlenza OV. Comprometimento cognitivo leve. Rev Med 2015;94:162-8. https://doi.org/10.11606/issn.16799836.v94i3p162-168

5.Dauncey MJ. Nutrition, the brain and cognitive decline: insights from epigenetics. Eur J Clin Nutr 2014;68:1179-85. https://doi.org/10.1038/ejcn.2014.173

6. Wang $\mathrm{H}$, Cao G, Prior RL. Total antioxidant capacity of fruits. J Agricult Food Chem

$1996 ; 44: 701-5$. https://doi.org/10.1021/jf950579y

7.Sun J, Chu YF, Wu X, Liu RH. Antioxidant and antiproliferative activities of common fruits. J Agric Food Chem 2002;50:7449-54. https://doi.org/10.1021/jf0207530

8. Wilson JX. Antioxidant defense of the brain: a role for astrocytes. Can J Physiol Pharm 1997;75:1149-63. https://doi.org/10.1139/cjpp-7510-11-1149 
9. Halliwell B, Gutteridge JM. Free radicals in biology and medicine. New York: Oxford University Press. 2015, p.896. https://doi.org/10.1093/acprof:oso/9780198717478.001.0001

10.Evans $\mathrm{PH}$. Free radicals in brain metabolism and pathology. Bri Med Bull 1993;49:577-87.

https://doi.org/10.1093/oxfordjournals.bmb.a072632

11.Gibson EL, Green MW. Nutritional influences on cognitive function: mechanisms of susceptibility. Nutr Res Rev 2002;15:169-206. https://doi.org/10.1079/NRR200131

12. Cardoso BR, Bandeira VS, Jacob-Filho W, Cozzolino SMF. Selenium status in elderly: relation to cognitive decline. J Trace Elem Med Biol 2014;28:422-6. https://doi.org/10.1016/j.jtemb.2014.08.009

13. Cardoso BR, Apolinário D, da Silva Bandeira V, Busse AL, Magaldi RM, Jacob-Filho W, et al. Effects of Brazil nut consumption on selenium status and cognitive performance in older adults with mild cognitive impairment: a randomized controlled pilot trial. Eur J Nutr 2016;55:107-16. https://doi.org/10.1007/s00394-014-0829-2

14.Cechella JL, Leite MR, Pinton S, Zeni G, Nogueira CW. Neuroprotective benefits of aerobic exercise and organoselenium dietary supplementation in hippocampus of old rats. Molecul Neurobiol 2018;55:3832-40. https://doi.org/10.1007/s12035-017-0600-9

15.Cechella JL, Leite MR, Gai RM, Zeni G. The impact of a diphenyl diselenide-supplemented diet and aerobic exercise on memory of middle-aged rats. Physiol Behav 2014;135:125-9. https://doi.org/10.1016/j.physbeh.2014.06.006

16.Cechella JL, Leite MR, Rosario AR, Sampaio TB, Zeni G. Diphenyl diselenide-supplemented diet and swimming exercise enhance novel object recognition memory in old rats. Age 2014;36:9666. https://doi.org/10.1007/s11357-014-9666-8

17.Demirci K, Nazıroğlu M, Övey İS, Balaban H. Selenium attenuates apoptosis, inflammation and oxidative stress in the blood and brain of aged rats with scopolamine-induced dementia. Metabol Brain Dis 2017;32:321-9. https://doi.org/10.1007/s11011-016-9903-1

18. Balaban H, Nazıroğlu M, Demirci K, Övey İS. The protective role of selenium on scopolamine-induced memory impairment, oxidative stress, and apoptosis in aged rats: the involvement of TRPM2 and TRPV1 channels. Molecul Neurobiol 2017;54:2852-68. https://doi.org/10.1007/s12035-016-9835-0

19.Zhang $\mathrm{ZH}, \mathrm{Wu} \mathrm{QY}$, Zheng $\mathrm{R}$, Chen $\mathrm{C}$, Chen $\mathrm{Y}$, Liu Q, et al. Selenomethionine mitigates cognitive decline by targeting both tau hyperphosphorylation and autophagic clearance in an Alzheimer's disease mouse model. J Neurosci 2017;37:2449-62. https://doi.org/10.1523/JNEUROSCI.3229-16.2017

20.McKhann G, Drachman D, Folstein M, Katzman R, Price D, Stadlan EM. Clinical diagnosis of Alzheimer's disease: report of the NINCDSADRDA work group under the auspices of Department of Health and Human Services Task Force on Alzheimer's disease. Neurology 1984;34:939-44. https://doi.org/10/1212/WNL.34.7.939 
21. Winblad B, Palmer K, Kivipelto M, Jelic V, Fratiglioni L, Wahlund LO, et al. Mild cognitive impairment - beyond controversies, towards a consensus: report of the International Working Group on Mild Cognitive Impairment. J Int Med 2004;256:240-6. https://doi.org/10.1111/j.1365-2796.2004.01380.x

22. Hao DQ, Xie GH, Zhang YM, Tian GJ. Determination of serum selenium by hydride generation flame atomic absorption spectrometry. Talanta 1996;43:595-600. https://doi.org/10.1016/00399140(95)01786-0

23.Behr CS. Efeito de uma dieta enriquecida com castanha-do-brasil (Bertholletia excelsa, L.) no estado nutricional relativo ao selênio de idosos não institucionalizados (Tese). São Paulo: Universidade de São Paulo, 2004.

24.Fagundes DJ, Taha MO. Animal disease model: choice's criteria and current animals specimens. Acta Cir Bras 2004;19:59-65. https://doi.org/10.1590/S0102-86502004000100010

25. Andreollo NA, Santos EFD, Araújo MR, Lopes LR. Idade dos ratos versus idade humana: qual é a relação? Arq Bras Cirur Dig 2012;25:49-51. https://doi.org/10.1590/S010267202012000100011

26. Ravi Kiran T, Subramanyam MVV, Devi SA. Swim exercise training and adaptations in the antioxidant defense system of myocardium of old rats: relationship to swim intensity and duration. Comp Biochem Physiol B Biochem Mol Biol 2004;137:187-96. https://doi.org/10.1016/j.cbpc.2003.11.002

27.Cambras T, Diez-Noguera A. Evolution of rat motor activity circadian rhythm under three different light patterns. Physiol Behav 1991;49:63-8. https://doi.org/10.1016/0031-9384(91)90231C

28. Rosa RM, Flores DG, Appelt HR, Braga AL, Henriques JA, Roesler R. Facilitation of long-term object recognition memory by pretraining administration of diphenyl diselenide in mice. Neurosci Lett 2003;341:217-20. https://doi.org/10.1016/s0304-3940(03)00187-3 29.Souza AC, Bruning CA, Leite MR, Zeni G, Nogueira CW. Diphenyl diselenide improves scopolamine-induced memory impairment in mice. Behav Pharmacol https://doi.org/10.1097/FBP.0b013e32833befcf

30.Stangherlin EC, Luchese C, Pinton S, Rocha JB, Nogueira CW. Subchronical exposure to diphenyl diselenide enhances acquisition and retention of spatial memory in rats. Brain Res 2008;1201:106-13. https://doi.org/10.1016/j.brainres.2008.01.061

31. Maciel EN, Flores EM, Rocha JB, Folmer V. Comparative deposition of diphenyl diselenide in liver, kidney, and brain of mice. Bull Environ Contam Toxicol 2003;70:470-6. https://doi.org/10.1007/s00128-0030010-8

32.Bartus RT. On neurodegenerative diseases, models, and treatment strategies: lessons learned and lessons forgotten a generation following the cholinergic hypothesis. Exp Neurol 2000;163:495-529. https://doi.org/10.1006/exnr.2000.7397 
33.Pascale A, Etcheberrigaray R. Calcium alterations in Alzheimer's disease: pathophysiology, models and therapeutic opportunities. Pharmacol Res 1999;39:81-8.

https://doi.org/10.1006/phrs.1998.0411

34.Parajuli $B$, Sonobe $Y$, Horiuchi $H$, Takeuchi $H$, Mizuno $T$, Suzumura A. Oligomeric amyloidßinduces IL-1ßprocessing via production of ROS: implication in Alzheimer's disease. Cell Death Dis 2013;4:e975. https://doi.org/10.1038/cddis.2013.503

35.Soares RO, Horiquini-Barbosa E, Almeida SS, Lachat JJ. Environmental enrichment protects spatial learning and hippocampal neurons from the long-lasting effects of protein malnutrition early in life. Behav Brain Res 2017;335:55-62. https://doi.org/0.1016/j.bbr.2017.08.012 36.Song G, Zhang Z, Wen L, Chen C, Shi Q, Zhang $Y$, et al. Selenomethionine ameliorates cognitive decline, reduces tau hyperphosphorylation, and reverses synaptic deficit in the triple transgenic mouse model of Alzheimer's disease. J Alzheimers Dis 2014;41:85-99. https://doi.org/10.3233/JAD-131805 